\title{
Cytomegalovirus reactivation in inflammatory bowel disease: an uncommon occurrence related to corticosteroid dependence
}

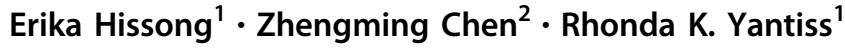

Received: 28 November 2018 / Revised: 30 January 2019 / Accepted: 31 January 2019 / Published online: 5 April 2019

(c) United States \& Canadian Academy of Pathology 2019

\begin{abstract}
Cytomegalovirus promotes mucosal injury in patients with inflammatory bowel disease, historically affecting $10-25 \%$ of ulcerative colitis patients with refractory disease. Viral reactivation is likely related to long-term corticosteroid therapy, which is no longer central to maintenance of patients with inflammatory bowel disease. We hypothesize that viral detection rates have decreased in the modern era, reflecting widespread use of immunomodulatory agents to control inflammation. We performed this study to evaluate the relationships between medical regimens and cytomegalovirus detection rates among patients with inflammatory bowel disease. We searched our database for all patients with established inflammatory bowel disease and severe flares diagnosed from 2002 to 2017. Patients maintained with corticosteroid therapy were considered to be corticosteroid-dependent and those treated with other agents were classified as corticosteroid-independent, provided they had not received corticosteroids within 6 months of colonoscopy. Biopsy samples were reviewed for viral inclusions and subjected to cytomegalovirus immunohistochemistry, and rates of viral detection were compared between groups. There were 135 corticosteroid-dependent patients; most had ulcerative colitis flares occurring during the 2002-2009 period. Patients with ulcerative colitis and Crohn disease were equally represented in the corticosteroid-independent group $(n=133)$ and most were evaluated for disease flares during the 2010-2017 interval. Cytomegalovirus was detected in 13 (8\%) cases; 9 (69\%) were diagnosed from 2002 to 2009 and all were obtained from corticosteroid-dependent patients $(p=<0.001)$. We conclude that rates of cytomegalovirus-related enterocolitis are declining among inflammatory bowel disease patients, reflecting a shift away from corticosteroid-based maintenance therapy in favor of more effective agents that do not promote viral reactivation.
\end{abstract}

\section{Introduction}

Cytomegalovirus is a member of the human herpesvirus family with a high prevalence of infection among adult patients $[1,2]$. Although primary infection often produces no, or only mild symptoms, reactivation of latent disease

Support: Translational Research Program, Department of Pathology and Laboratory Medicine, Weill Cornell Medicine

Erika Hissong

emh9016@med.cornell.edu

1 Department of Pathology and Laboratory Medicine, Weill Cornell Medicine, New York, NY, USA

2 Division of Biostatistics and Epidemiology, Department of Healthcare Policy and Research, Weill Cornell Medicine, New York, NY, USA is a common cause of systemic illness among immunocompromised patients. Risk is highest among patients with HIV/AIDS and transplant recipients but is also increased among individuals receiving systemic chemotherapy, immunosuppressive treatment for immunemediated diseases, those who are pregnant, and elderly patients with no other apparent causes of immunodeficiency [3-6]. Reactivation of cytomegalovirus in the gastrointestinal tract is a well-known complication of immunosuppressive therapy among patients with inflammatory bowel disease, in which case symptoms and histologic features often mimic a flare of disease activity. The incidence of cytomegalovirus reactivation in this setting ranges from $4.5 \%$ to $16 \%$, although some data suggest that up to $25 \%$ of patients with chronic, medically refractory colitis harbor cytomegalovirus in inflamed mucosae [7-14]. Distinction between cytomegalovirusrelated inflammation and a flare of inflammatory bowel disease is clinically important. Failure to recognize 
cytomegalovirus as the cause of symptoms generally leads clinicians to conclude that the level of immunosuppression is inadequate. Increased immunosuppression can worsen cytomegalovirus-induced inflammation, causing tissue necrosis and even bowel perforation. Unfortunately, detection of viral inclusions in the setting of inflammatory bowel disease is challenging because infected cells are often scarce or obscured by mucosal inflammation $[15,16]$. Thus, many pathologists routinely perform immunohistochemical stains to facilitate cytomegalovirus detection in samples from patients with inflammatory bowel disease.

Although it is our practice to obtain cytomegalovirus immunostains whenever biopsy samples from treated inflammatory bowel disease patients show chronic active enterocolitis and ulcers, we have noted a striking decrease in the number of immunopositive cases over the past several years. We believe this observation is related to decreased use of corticosteroids as maintenance therapy for these patients. We performed the current study to determine the frequency of cytomegalovirus detection among inflammatory bowel disease patients treated in an era of widespread use of immunomodulatory and biologic agents for both ulcerative colitis and Crohn disease and compared the results with historical data. We identified colonic biopsy samples from inflammatory bowel disease patients with chronic active colitis and ulcers treated during a 15-year period (2002-2017) and evaluated them for the presence of cytomegalovirus using a combination of histologic review and immunohistochemistry. Rates of viral detection among patients maintained with corticosteroid therapy were compared with those of patients treated with a combination of aminosalicylates, biologic agents, and immunomodulatory drugs, in order to determine whether modern therapies have affected rates of cytomegalovirus reactivation in patients with inflammatory bowel disease.

\section{Materials and methods}

\section{Case selection}

We retrospectively reviewed the files of the Department of Pathology and Laboratory Medicine at Weill Cornell Medicine to identify all cases of chronic colitis and ulcers diagnosed from 2002 to 2017, inclusively. Electronic medical records of 579 patients were reviewed and information regarding the nature of disease, duration of colitis, type and duration of medical treatment, and colectomy status was recorded. Untreated patients with newly diagnosed inflammatory bowel disease, patients with prior surgery, and individuals with chronic colitis due to other causes were excluded. Patients were classified as either corticosteroid-dependent or corticosteroid-independent. Corticosteroid-dependent patients received corticosteroids in their maintenance regimens and were regularly taking corticosteroids at the time of colonoscopy. Patients were considered to be corticosteroid-independent when they were maintained with various combinations of other drugs, such as aminosalicylates, immunomodulators, and biologic agents. Patients who had recently ( $<6$ months) transitioned from corticosteroid-based regimens to other agents, or received corticosteroids for symptom flares within 6 months of colonoscopy, were excluded from the study in order to limit potential confounders.

\section{Histopathologic evaluation}

Routinely processed, hematoxylin and eosin (H\&E)-stained tissue sections from all cases were evaluated for cytomegalovirus inclusions. Samples with large atypical cells that showed viral cytopathic changes (e.g. nucleomegaly, granular cytoplasmic inclusions, and/or nuclear inclusions) were classified as positive for cytomegalovirus and confirmed with immunohistochemical stains (DAKO cytomegalovirus M0854 Santa Clara, CA) using standard techniques and appropriate controls. All other samples with severely active chronic colitis and ulcers were also subjected to immunohistochemistry. Viral detection rates among corticosteroiddependent patients were compared with those of patients receiving other therapeutic agents. Fisher's exact test was used to test the associations among categorical variables. Firth's penalized likelihood method was used for multivariable logistic regression. All analyses were performed using statistical software SAS Version 9.4 (SAS Institute, Cary, NC). A $p$ value $<0.05$ was considered to be statistically significant.

\section{Results}

The study group consisted of 298 biopsy samples from 268 patients, including 169 with ulcerative colitis and 99 with Crohn disease. Their clinical features are summarized in Table 1. Colonic biopsy samples were obtained from 268 patients, including 115 men and 153 women. There were 135 patients in the corticosteroid-dependent group and 133 corticosteroid-independent patients. Individuals in the latter group were maintained with aminosalicylates, biologic agents (e.g. infliximab, adalimumab), immunomodulatory drugs (e.g. methotrexate, mercaptopurine), or a combination of multiple agents. Most $(78 \%)$ patients in the corticosteroid-dependent group had underlying ulcerative colitis, whereas similar numbers of patients in the corticosteroid-independent group had 
Table 1 Clinicopathologic features of corticosteroid-dependent inflammatory bowel disease patients and those maintained with other therapies

\begin{tabular}{lll}
\hline & $\begin{array}{l}\text { Corticosteroid- } \\
\text { dependent patients } \\
(N=135)\end{array}$ & $\begin{array}{l}\text { Corticosteroid- } \\
\text { independent patients } \\
(N=133)\end{array}$ \\
\hline $\begin{array}{lll}\text { Age (mean) } \\
\text { Male/female ratio }\end{array}$ & $\begin{array}{l}\text { 46 years } \\
\text { 5iagnosis }\end{array}$ & $\begin{array}{l}43 \text { years } \\
63 / 70\end{array}$ \\
$\begin{array}{l}\text { Crohn disease } \\
\text { Ulcerative colitis }\end{array}$ & $30(22 \%)$ & $69(52 \%)$ \\
Tissue biopsy collection & $64(48 \%)$ \\
2002-2009 & $102(76 \%)$ & $2(2 \%)$ \\
2010-2017 & $33(24 \%)$ & $131(98 \%)$ \\
$\begin{array}{l}\text { Cytomegalovirus } \\
\text { detection }\end{array}$ & $12(9 \%)$ & $0(0 \%)$ \\
2002-2009 & $8(67 \%)$ & $0(0 \%)$ \\
2010-2017 & $4(33 \%)$ & $0(0 \%)$ \\
Subsequent & $38(28 \%)$ & $20(15 \%)$ \\
colectomy & &
\end{tabular}

ulcerative colitis (48\%) and Crohn disease (52\%). Most patients in both groups were middle-aged adults with a slight female predominance. Not surprisingly, $76 \%$ of corticosteroid-dependent patients were examined prior to 2010 and most $(83 \%)$ had underlying ulcerative colitis. Patients with ulcerative colitis $(58 \%)$ and Crohn disease (75\%) were more likely to be managed with immunomodulatory therapies and/or biologic agents after 2010 . Treatment regimens for patients in the corticosteroidindependent group included aminosalicylates alone $(n=$ $43)$, immunomodulatory agents alone $(n=17)$, biologic agents alone $(n=44)$, and a combination of immunomodulatory and biologic agents $(n=29)$. Thirty-eight patients $(28 \%)$ in the corticosteroid-dependent group and 20 patients $(15 \%)$ in the corticosteroid-independent group ultimately underwent a colectomy procedure $(p=0.01)$.

Cytomegalovirus inclusions were detected in 13 (8\%) biopsy samples from 12 patients. They were detected in routinely stained sections in 12 samples and one sample contained enlarged, atypical endothelial cells suspicious for cytomegalovirus infection. All of these cases were confirmed with cytomegalovirus immunohistochemistry (Fig. 1). The number of inclusions present in each case ranged from a single cell to more than ten infected cells per sample. Patients with cytomegalovirus reactivation were older than cytomegalovirus-negative individuals (mean: 63 years versus 44 years, $p=<0.01$ ) and tended to be women, although the sex difference was not significant $(p=0.08)$. Ten $(83 \%)$ patients with viral inclusions in their biopsy samples had ulcerative colitis; the duration of disease at the time of detection ranged from several months to 30 years.
All patients with detectable cytomegalovirus were corticosteroid-dependent and had received corticosteroid therapy within 2 months of the endoscopic procedure. Serologic studies were performed for three patients with biopsy-proven cytomegalovirus infection; all had elevated anti-cytomegalovirus IgG titers, but anti-cytomegalovirus IgM was not detected in any patient (Table 2). Quantitative PCR for cytomegalovirus was performed on peripheral blood from six patients with cytomegalovirus-positive biopsy samples. Viral loads ranged from $<200$ copies $/ \mathrm{mL}$ to 63,600 copies $/ \mathrm{mL}$; there was no correlation between the viral load and density of viral inclusions in colonic samples. Seven $(58 \%)$ patients with cytomegalovirus reactivation eventually underwent colectomy, which was a significantly higher rate than that of the rest of the cohort $(22 \%, p<$ 0.05 ), and higher than that of corticosteroid-dependent patients with cytomegalovirus-negative colonic samples $(25 \%, p=0.04)$. Multivariable logistic regression demonstrated that cytomegalovirus detection was significantly associated with corticosteroid use, independent of age and sex $(p=0.03)$. A sub-analysis limited to ulcerative colitis patients revealed a significant relationship between corticosteroid use and cytomegalovirus detection $(p=0.014)$. Of note, most (69\%) cytomegalovirus-positive cases were obtained during the 2002-2009 period. Rates of viral detection declined from $7.4 \%$ (2002-2009) to $2.2 \%$ (2010-2017) during the study period.

\section{Discussion}

The purpose of this study was to systematically evaluate relationships between treatment regimens and cytomegalovirus detection rates among patients with established inflammatory bowel disease and to re-evaluate the role of cytomegalovirus immunohistochemistry in an era of immunomodulatory and biologic agents. We performed cytomegalovirus immunohistochemistry on 298 samples from 268 patients with established inflammatory bowel disease and ulcers despite medical therapy. We found that cytomegalovirus detection is strongly associated with recent corticosteroid use $(p<0.001)$, particularly among patients with ulcerative colitis $(p=0.02)$, and these relationships persist following multivariable logistic regression analysis. We also found that rates of cytomegalovirus detection have decreased from $7.4 \%$ to $2.2 \%$ over a 15 -year period, corresponding with an overall decline in corticosteroid use among both ulcerative colitis and Crohn disease patients. These findings suggest that cytomegalovirus is an unlikely cause of colitic symptoms among inflammatory bowel disease patients who have not recently received corticosteroid therapy. Routine use of cytomegalovirus immunohistochemical stains in all inflammatory bowel disease patients 


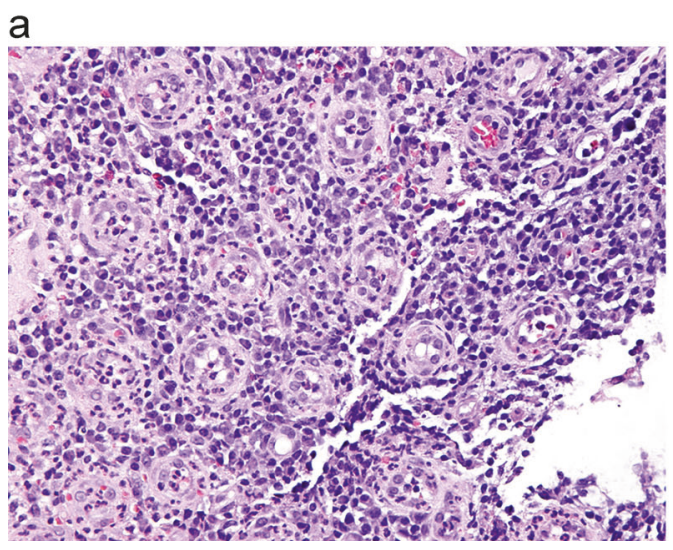

Fig. 1 Twelve patients had histologically evident, immunohistochemically confirmed cytomegalovirus in their colonic biopsy samples. One biopsy sample showed ulcers and inflamed granulation tissue without

Table 2 Results of serologic studies from patients with cytomegalovirus inclusions in colonic biopsy samples

\begin{tabular}{lll}
\hline Patient & Viral load (DNA copies/mL) & Serologic findings \\
\hline 1 & - & - \\
2 & - & - \\
3 & 63,600 & - \\
4 & - & - \\
5 & - & - \\
6 & - & - \\
7 & $<200$ & - \\
8 & 900 & $\mathrm{IgG}+/ \mathrm{IgM}-$ \\
9 & - & $\mathrm{IgG}+/ \mathrm{IgM}-$ \\
10 & $<200$ & $\mathrm{IgG}+/ \mathrm{IgM}-$ \\
11 & 1818 & - \\
12 & $<137$ & - \\
\hline
\end{tabular}

with medically refractory disease may be unnecessary in the modern era.

Cytomegalovirus reactivation is a well-known complication of inflammatory bowel disease treatment and distinguishing it from an exacerbation of existing inflammatory bowel disease is clinically important. Additional immunosuppressive therapy usually worsens cytomegalovirusrelated intestinal injury and can result in toxic megacolon, perforation, or even death [17-19]. Reported frequencies of cytomegalovirus reactivation in the setting of inflammatory bowel disease vary depending on the method of viral detection, nature of underlying inflammatory bowel disease, severity of inflammatory activity, and type of therapeutic intervention $[7,8,18,20,21]$. The diagnosis of cytomegalovirus-related colonic inflammation largely rests on histologic identification of viral inclusions in tissue samples, which can be enhanced with immunohistochemistry. Serologic studies are of limited value because they do

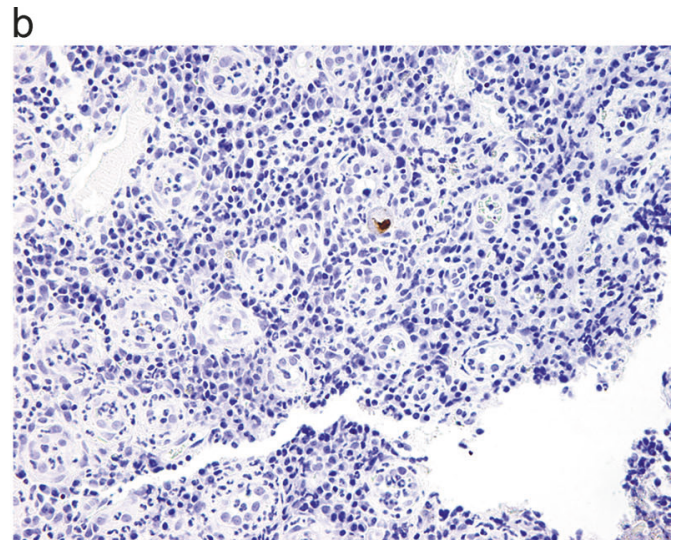

detectable cytomegalovirus inclusions (a). However, occasional enlarged endothelial cells strong nuclear immunostaining for cytomegalovirus in the same sample (b)

not always distinguish remote viral exposure from active infection. Assays that detect cytomegalovirus DNA by PCR are frequently performed to detect symptomatic cytomegalovirus infection but do not necessarily correlate with the abundance of inclusions detected in mucosal samples [22].

Reactivation of cytomegalovirus is more likely among patients with inflammatory bowel disease who are further immunocompromised by poor nutrition, medications, or immune dysfunction. The virus demonstrates tropism for sites of inflammation and, thus, reactivation risk is often related to extent and severity of inflammation. Overexpression of tumor necrosis factor (TNF) promotes disease activity and plays a role in activating latent virus through interactions with other proinflammatory cytokines [23-28]. Presumably, circulating monocytes and dendritic cells that contain latent cytomegalovirus migrate to sites of inflammation, where they elaborate IL-6, TNF- $\alpha$, IL-1 $\beta$, and other cytokines [23, 29-31]. Yi et al. utilized a combination of serum PCR, tissue samples, and antibody titers to evaluate the prevalence of, and clinical risk factors for, cytomegalovirus infection among 189 ulcerative colitis patients and 37 with Crohn disease; they found elevated serum DNA levels were associated with severe disease activity $(p=0.05)$ and recent corticosteroid therapy $(p=0.04)$ [7]. Others have also found a higher prevalence of cytomegalovirus reactivation among patients with refractory ulcerative colitis, likely reflecting the tendency for corticosteroids to directly activate viral replication and suppress antiviral T-cell specific function [8, 12, 13, 18, 32-35].

Therapeutic management of inflammatory bowel disease has drastically changed over the last several years. Corticosteroids are mostly reserved for induction of remission but are not widely used for maintenance therapy due to a variety of adverse effects [36]. Single immunomodulatory and/or biologic agents, or combinations of drugs, are highly effective for maintaining remission and 
preventing relapse among both ulcerative colitis and Crohn disease patients. Azathioprine, 6-mercaptopurine, and methotrexate reduce recurrence rates of both Crohn disease and ulcerative colitis among patients with mild to moderate disease [37-41]. Biological agents, including infliximab, adalimumab, and certolizumab pegol, inhibit TNF- $\alpha$-mediated inflammation and are effective treatment options for patients with moderate-to-severe ulcerative colitis and Crohn disease [42-44]. Cyclophosphamide, cyclosporine, and tacrolimus can also be used in selected patients.

The reported prevalence of infectious complications among patients receiving immunomodulatory and biologic therapies varies considerably. Early randomized clinical trials reported no increased risk for serious infections among patients treated with TNF- $\alpha$ inhibitors, yet subsequent studies have reported infection rates of $2.8-4 \%$ among patients receiving biologic agents [45-50]. Commonly reported infections include hepatitis B virus, herpes zoster virus, and tuberculosis reactivation [51-54]. Overall, the odds of developing an infection while receiving biologic agents are increased (19\%), but numerous placebo-controlled trials have failed to consistently demonstrate a risk of serious infection [55]. Cytomegalovirus reactivation is less commonly associated with newer agents than corticosteroid therapy [56-60]. McCurdy et al. performed a retrospective case-control study and found that medically refractory inflammatory bowel disease (OR 3.69), corticosteroid therapy (OR 2.95), and immunomodulatory drug therapy (OR 1.86) were significantly associated with cytomegalovirusrelated colitis whereas TNF- $\alpha$ antagonists did not cause cytomegalovirus-related morbidity [20]. Doménech et al. performed a prospective study evaluating 114 patients with ulcerative colitis and identified cytomegalovirus colitis in only patients who had received high doses of corticosteroids within 7-10 days of the diagnostic procedure [8].

In summary, our findings indicate that the rate of cytomegalovirus detection among patients with colitic symptoms is declining among patients with established inflammatory bowel disease. This trend likely reflects a shift away from maintenance therapy with corticosteroids toward more effective biologic and immunomodulatory agents, particularly among patients with ulcerative colitis. Immunohistochemical stains for cytomegalovirus are of limited value when applied to samples obtained from patients with inflammatory bowel disease who have not been recently exposed to corticosteroid therapy, especially when they are maintained with biologic agents.

\section{Compliance with ethical standards}

Conflict of interest The authors declare that they have no conflict of interest.
Publisher's note: Springer Nature remains neutral with regard to jurisdictional claims in published maps and institutional affiliations.

\section{References}

1. Krech U. Complement-fixing antibodies against cytomegalovirus in different parts of the world. Bull World Health Organ. 1973;49:103-6.

2. de la Hoz RE, Stephens G, Sherlock C. Diagnosis and treatment approaches of cytomegalovirus infections in adult patients. J Clin Virol. 2002;25(Suppl 2):S1-12.

3. Meyers JD, Flournoy N, Thomas ED. Risk factors for cytomegalovirus infection after human marrow transplantation. J Infect Dis. 1986;153:478-88.

4. de Jong MD, Galasso GJ, Gazzard B, et al. Summary of the II international symposium on cytomegalovirus. Antivir Res. 1998;39:141-62.

5. Goodgame RW. Gastrointestinal cytomegalovirus disease. Ann Intern Med. 1993;119:924-35.

6. Olsson J, Wikby A, Johansson B, et al. Age-related change in peripheral blood T-lymphocyte subpopulations and cytomegalovirus infection in the very old: the Swedish longitudinal OCTO immune study. Mech Ageing Dev. 2000; 121:187-201.

7. Yi F, Zhao J, Luckheeram RV, et al. The prevalence and risk factors of cytomegalovirus infection in inflammatory bowel disease in Wuhan, Central China. Virol J. 2013;10:43.

8. Domenech E, Vega R, Ojanguren I, et al. Cytomegalovirus infection in ulcerative colitis: a prospective, comparative study on prevalence and diagnostic strategy. Inflamm Bowel Dis. 2008;14:1373-9.

9. Kim YS, Kim YH, Kim JS, et al. The prevalence and efficacy of ganciclovir on steroid-refractory ulcerative colitis with cytomegalovirus infection: a prospective multicenter study. J Clin Gastroenterol. 2012;46:51-6.

10. Berk T, Gordon SJ, Choi HY, et al. Cytomegalovirus infection of the colon: a possible role in exacerbations of inflammatory bowel disease. Am J Gastroenterol. 1985;80:355-60.

11. Leveque N, Brixi-Benmansour $\mathrm{H}$, Reig $\mathrm{T}$, et al. Low frequency of cytomegalovirus infection during exacerbations of inflammatory bowel diseases. J Med Virol. 2010;82:1694-700.

12. Kambham N, Vij R, Cartwright CA, et al. Cytomegalovirus infection in steroid-refractory ulcerative colitis: a case-control study. Am J Surg Pathol. 2004;28:365-73.

13. Kim JJ, Simpson N, Klipfel N, et al. Cytomegalovirus infection in patients with active inflammatory bowel disease. Dig Dis Sci. 2010;55:1059-65.

14. Criscuoli V, Casa A, Orlando A, et al. Severe acute colitis associated with cytomegalovirus: a prevalence study. Dig Liver Dis. 2004;36:818-20.

15. Kandiel A, Lashner B. Cytomegalovirus colitis complicating inflammatory bowel disease. Am J Gastroenterol. 2006;101: 2857-65.

16. Beaugerie L, Cywiner-Golenzer C, Monfort L, et al. Definition and diagnosis of cytomegalovirus colitis in patients infected by human immunodeficiency virus. J Acquir Immune Defic Syndr Hum Retrovirol. 1997;14:423-9.

17. Cooper HS, Raffensperger EC, Jonas L, et al. Cytomegalovirus inclusions in patients with ulcerative colitis and toxic dilation requiring colonic resection. Gastroenterology. 1977;72:1253-6.

18. Romkens TE, Bulte GJ, Nissen LH, et al. Cytomegalovirus in inflammatory bowel disease: a systematic review. World J Gastroenterol. 2016;22:1321-30.

19. Iida $\mathrm{T}$, Ikeya $\mathrm{K}$, Watanabe $\mathrm{F}$, et al. Looking for endoscopic features of cytomegalovirus colitis: a study of 187 patients with 
active ulcerative colitis, positive and negative for cytomegalovirus. Inflamm Bowel Dis. 2013;19:1156-63.

20. McCurdy JD, Jones A, Enders FT, et al. A model for identifying cytomegalovirus in patients with inflammatory bowel disease. Clin Gastroenterol Hepatol. 2015;13:131-7. quize7

21. Dieterich DT, Rahmin M. Cytomegalovirus colitis in AIDS: presentation in 44 patients and a review of the literature. J Acquir Immune Defic Syndr. 1991;4(Suppl 1):S29-35.

22. Wethkamp N, Nordlohne EM, Meister V, et al. Identification of clinically relevant cytomegalovirus infections in patients with inflammatory bowel disease. Mod Pathol. 2018;31:527-38.

23. Soderberg-Naucler C, Fish KN, Nelson JA. Interferon-gamma and tumor necrosis factor-alpha specifically induce formation of cytomegalovirus-permissive monocyte-derived macrophages that are refractory to the antiviral activity of these cytokines. J Clin Investig. 1997;100:3154-63.

24. Simon CO, Seckert CK, Dreis D, et al. Role for tumor necrosis factor alpha in murine cytomegalovirus transcriptional reactivation in latently infected lungs. J Virol. 2005;79:326-40.

25. Fietze E, Prosch S, Reinke P, et al. Cytomegalovirus infection in transplant recipients. The role of tumor necrosis factor. Transplantation. 1994;58:675-80.

26. Matsumura K, Nakase H, Kosugi I, et al. Establishment of a novel mouse model of ulcerative colitis with concomitant cytomegalovirus infection: in vivo identification of cytomegalovirus persistent infected cells. Inflamm Bowel Dis. 2013;19:1951-63.

27. Docke WD, Prosch S, Fietze E, et al. Cytomegalovirus reactivation and tumour necrosis factor. Lancet. 1994;343:268-9.

28. Nakase H, Yoshino T, Honzawa $\mathrm{Y}$, et al. Low prevalence of cytomegalovirus infection in patients with Crohn's disease in comparison with ulcerative colitis: effect of different immune response on prevalence of cytomegalovirus infection. Dig Dis Sci. 2010;55:1498-9.

29. Chan G, Bivins-Smith ER, Smith MS, et al. Transcriptome analysis reveals human cytomegalovirus reprograms monocyte differentiation toward an M1 macrophage. J Immunol. 2008; 181:698-711.

30. Soderberg-Naucler C, Nelson JY. Human cytomegalovirus latency and reactivation: a delicate balance between the virus and its host's immune system. Intervirology. 1999;42:314-21.

31. Hahn G, Jores R, Mocarski ES. Cytomegalovirus remains latent in a common precursor of dendritic and myeloid cells. Proc Natl Acad Sci USA. 1998;95:3937-42.

32. Wada $\mathrm{Y}$, Matsui $\mathrm{T}$, Matake $\mathrm{H}$, et al. Intractable ulcerative colitis caused by cytomegalovirus infection: a prospective study on prevalence, diagnosis, and treatment. Dis Colon Rectum. 2003;46: S59-65.

33. Widmann T, Sester U, Gartner BC, et al. Levels of cytomegalovirus specific CD4 $\mathrm{T}$ cells are dynamic and correlate with cytomegalovirus viremia after allogeneic stem cell transplantation. PLoS One. 2008;3:e3634.

34. Ninomiya E, Hattori T, Toyoda M, et al. Glucocorticoids promote neural progenitor cell proliferation derived from human induced pluripotent stem cells. + . 2014;3:527.

35. Cottone M, Pietrosi G, Martorana G, et al. Prevalence of cytomegalovirus infection in severe refractory ulcerative and Crohn's colitis. Am J Gastroenterol. 2001;96:773-5.

36. Matsuoka K, Kobayashi T, Ueno F, et al. Evidence-based clinical practice guidelines for inflammatory bowel disease. J Gastroenterol. 2018;53:305-53.

37. Lichtenstein GR, Abreu MT, Cohen R, et al. American Gastroenterological Association Institute medical position statement on corticosteroids, immunomodulators, and infliximab in inflammatory bowel disease. Gastroenterology. 2006;130:935-9.

38. Khan KJ, Dubinsky MC, Ford AC, et al. Efficacy of immunosuppressive therapy for inflammatory bowel disease: a systematic review and meta-analysis. Am J Gastroenterol. 2011; 106:630-42.

39. Prefontaine E, Sutherland LR, Macdonald JK, et al. Azathioprine or 6-mercaptopurine for maintenance of remission in Crohn's disease. Cochrane Database Syst Rev. 2009; CD000067. https://doi.org/10.1002/14651858.CD000067.

40. Feagan BG, Fedorak RN, Irvine EJ, et al. A comparison of methotrexate with placebo for the maintenance of remission in Crohn's disease. North American Crohn's Study Group Investigators. N Engl J Med. 2000;342:1627-32.

41. Feagan BG, Rochon J, Fedorak RN, et al. Methotrexate for the treatment of Crohn's disease. The North American Crohn's Study Group Investigators. N Engl J Med. 1995;332:292-7.

42. Sandborn WJ, Feagan BG. The efficacy of azathioprine and 6-mercaptopurine for the prevention of postoperative recurrence in patients with Crohn's disease remains uncertain. Gastroenterology. 2004;127:990-3.

43. Reinecker HC, Steffen M, Witthoeft T, et al. Enhanced secretion of tumour necrosis factor-alpha, IL-6, and IL- 1 beta by isolated lamina propria mononuclear cells from patients with ulcerative colitis and Crohn's disease. Clin Exp Immunol. 1993;94:174-81.

44. Reimund JM, Wittersheim C, Dumont S, et al. Mucosal inflammatory cytokine production by intestinal biopsies in patients with ulcerative colitis and Crohn's disease. J Clin Immunol. 1996; 16:144-50.

45. Clark M, Colombel JF, Feagan BC, et al. American gastroenterological association consensus development conference on the use of biologics in the treatment of inflammatory bowel disease, June 21-23, 2006. Gastroenterology. 2007;133:312-39.

46. Schreiber S, Rutgeerts P, Fedorak RN, et al. A randomized, placebocontrolled trial of certolizumab pegol (CDP870) for treatment of Crohn's disease. Gastroenterology. 2005;129:807-18.

47. Colombel JF, Sandborn WJ, Rutgeerts P, et al. Adalimumab for maintenance of clinical response and remission in patients with Crohn's disease: the CHARM trial. Gastroenterology. 2007; 132:52-65.

48. Sands BE, Anderson FH, Bernstein CN, et al. Infliximab maintenance therapy for fistulizing Crohn's disease. N Engl J Med. 2004;350:876-85.

49. Hanauer SB, Feagan BG, Lichtenstein GR, et al. Maintenance infliximab for Crohn's disease: the ACCENT I randomised trial. Lancet. 2002;359:1541-9.

50. Lichtenstein GR, Rutgeerts P, Sandborn WJ, et al. A pooled analysis of infections, malignancy, and mortality in infliximaband immunomodulator-treated adult patients with inflammatory bowel disease. Am J Gastroenterol. 2012;107:1051-63.

51. Rahier JF, Magro F, Abreu C, et al. Second European evidencebased consensus on the prevention, diagnosis and management of opportunistic infections in inflammatory bowel disease. J Crohns Colitis. 2014;8:443-68

52. Shale MJ, Seow CH, Coffin CS, et al. Review article: chronic viral infection in the anti-tumour necrosis factor therapy era in inflammatory bowel disease. Aliment Pharmacol Ther. 2010;31:20-34.

53. Askling J, Fored CM, Brandt L, et al. Risk and case characteristics of tuberculosis in rheumatoid arthritis associated with tumor necrosis factor antagonists in Sweden. Arthritis Rheumatol. 2005;52:1986-92.

54. Ryu HH, Lee EY, Shin K, et al. Hepatitis B virus reactivation in rheumatoid arthritis and ankylosing spondylitis patients treated with anti-TNFalpha agents: a retrospective analysis of 49 cases. Clin Rheumatol. 2012;31:931-6.

55. Bonovas S, Fiorino G, Allocca M, et al. Biologic therapies and risk of infection and malignancy in patients with inflammatory bowel disease: a systematic review and network meta-analysis. Clin Gastroenterol Hepatol. 2016;14:1385-97. e10 
56. Lavagna A, Bergallo M, Daperno M, et al. Infliximab and the risk of latent viruses reactivation in active Crohn's disease. Inflamm Bowel Dis. 2007;13:896-902.

57. Park SC, Jeen YM, Jeen YT. Approach to cytomegalovirus infections in patients with ulcerative colitis. Korean J Intern Med. 2017;32:383-92.

58. D'Ovidio V, Vernia P, Gentile G, et al. Cytomegalovirus infection in inflammatory bowel disease patients undergoing anti-TNFalpha therapy. J Clin Virol. 2008;43:180-3.
59. Criscuoli V, Mocciaro F, Orlando A, et al. Cytomegalovirus disappearance after treatment for refractory ulcerative colitis in 2 patients treated with infliximab and 1 patient with leukapheresis. Inflamm Bowel Dis. 2009;15:810-1.

60. Nakase H, Chiba T. TNF-alpha is an important pathogenic factor contributing to reactivation of cytomegalovirus in inflamed mucosa of colon in patients with ulcerative colitis: lesson from clinical experience. Inflamm Bowel Dis. 2010; $16: 550-1$. 